\title{
Enfermedades pulmonares en pacientes infectados con VIH hospitalizados en el Instituto Nacional del Tórax
}

\author{
Sara Chernilo S, Sergio Trujillo V, Mariana Kahn Cha, \\ Mónica Paredes P, G hislaine Echevarría 0 , \\ Claudia Sepúlveda L.
}

\section{Lung diseases among HIV infected patients admitted to the "Instituto N acional del Torax" in Santiago, Chile}

Background: Pulmonary diseases are common among HIV infected patients. The prevalence of the different diseases varies greatly. Aim: To identify the different pulmonary diseases that affect a Chilean population of HIV infected patients and to identify factors associated with in hospital mortality. Material and methods: Retrospective review of the clinical records of all HIV infected patients with lung diseases discharged from our institution during a period of 3.5 years. Collection of demographic and biomedical data. Results: One hundred seventy one patients (aged 35.7 years, 86\% men) had 236 episodes of lung diseases. Only $13.5 \%$ of the patients were receiving antiretroviral therapy and $18 \%$ were on pneumocystis prophylaxis. Infectious diseases accounted for $87 \%$ of the discharges, neoplasm for $5.1 \%$. Pneumocystis jirovecii infection was responsible for $37.7 \%$ of the episodes, community acquired pneumonia was seen in $24.1 \%$ and mycobacterial diseases in $14.4 \%$. Two or more conditions were present in $13.6 \%$. Death during hospital stay occurred in 19.5\%. Multivariate analysis identified pneumothorax as the only significant independent predictor of in-hospital mortality in patients with pneumocystis pneumonia, while nosocomial pneumonia was the only predictor of death among patients with non-pneumocystis pulmonary diseases. Conclusions: Infectious diseases were the main cause of hospitalization among Chilean HIV infected patients. Mortality among these patients remains high. Appropriate antiretroviral therapy and prophylaxis may alter pulmonary disease prevalence in the future. Every effort should be made to avoid the development of pneumothorax and nosocomial pneumonia (Rev Méd Chile 2005; 133: 517-24).

(Key Words: Antiretroviral therapy; HIV; Lung diseases; Respiratory tract infections)

Recibido el 15 de junio, 2004. Aceptado en versión corregida el 15 de marzo, 2005. Servicio Médico-Quirúrgico Instituto Nacional del Tórax.

aAlumna 5o año de Medicina.

Correspondencia a: Dra. Sara Chernilo S. Servicio MédicoQuirúrgico Instituto Nacional del Tórax. José Miguel Infante

\# 717, Santiago, Chile. E mail: sarachernilo@yahoo.es. 
$\mathrm{D}$ esde el inicio de la epidemia de SIDA, se reconoce a las enfermedades pulmonares como una causa importante de morbimortalidad en esta población ${ }^{1}$. Hasta $70 \%$ de los pacientes infectados con virus de inmunodeficiencia humana (VIH) pueden presentar una enfermedad pulmonar durante su evolución ${ }^{2}$. Existen estudios en autopsias que han demostrado compromiso del pulmón en 100\% de los casos a comienzos de la epidemia y en $84 \%$ de los pacientes que utilizan terapia antirretroviral altamente efectiva $^{2,3}$. El tipo de enfermedad pulmonar que se observa en una población de pacientes infectados con VIH depende del grado de inmunodepresión, del uso de terapia antirretroviral efectiva, uso de profilaxis específica y también de factores demográficos y geográficos $^{4}$. Es llamativa la elevada incidencia de neumonía por Pneumocystis jirovecii en pacientes norteamericanos y europeos, contrastando con lo que ocurre en Africa, donde esta neumonía es casi desconocida ${ }^{5,6}$. En nuestro país, Wolff y cols establecieron que la neumonía intersticial, presumiblemente producida por $\mathrm{P}$ jirovecii, y la tuberculosis eran las condiciones definitorias de SIDA más frecuentes en una cohorte de pacientes estudiados entre 1988 y 19937. En una publicación más reciente de este mismo grupo, las neumonías bacterianas fueron la enfermedad pulmonar más frecuente, alcanzando una incidencia de 18,6 casos por 100 pacientes/año en un grupo sin terapia antirretroviral ${ }^{8}$.

Identificar la patología pulmonar prevalente en pacientes chilenos infectados con VIH permitiría optimizar el uso de medidas profilácticas y el inicio precoz de esquemas terapéuticos empíricos que los beneficiarían directamente. El objetivo de este estudio retrospectivo fue identificar las enfermedades pulmonares que afectaban a los pacientes infectados con VIH que egresaron del Instituto Nacional del Tórax de Santiago de Chile en el período 1999-2003; asimismo, identificar los factores relacionados con la mortalidad en el hospital en estos pacientes.

\section{Material y Método}

Se revisaron los listados computacionales de todos los egresos hospitalarios del Instituto Nacional del Tórax de Santiago de Chile, entre el 1 de enero de 1999 y el 31 de julio de 2003.

El Instituto Nacional del Tórax, es un centro de referencia nacional en patología torácica dependien- te del Ministerio de Salud, dotado de 215 camas, que atiende preferentemente a beneficiarios del Sistema Nacional de Servicios de Salud. Los autores revisaron, siguiendo pautas preestablecidas, todas las fichas clínicas de los pacientes cuyo diagnóstico de egreso fuera infección por VIH, SIDA o diagnósticos relacionados. Los casos con dudas fueron resueltos consensuadamente por dos neumólogos.

Población. Durante el período del estudio egresaron del Instituto Nacional del Tórax 21.506 pacientes; 252 correspondieron a pacientes infectados con VIH. De éstos, se recuperaron 220 fichas correspondientes a 236 egresos. Los datos para los 16 egresos restantes se obtuvieron de 9 papeletas de egreso hospitalario, 5 certificados de defunción y una presentación a un congreso?. Los antecedentes demográficos, los diagnósticos de egreso que figuraban en la epicrisis y los resultados de exámenes de confirmación diagnóstica se registraron en una base de datos. Los diagnósticos de neumonía comunitaria ${ }^{10}$ y neumonía nosocomial $^{11}$ se realizaron según los criterios establecidos por las normas internacionales. Se consideró como confirmado al diagnóstico de egreso si cumplía las siguientes condiciones: neumonía si existió un cultivo de expectoración o lavado bronquial o lavado broncoalveolar o sangre positivos para un patógeno respiratorio; neumonía por $\mathrm{P}$ jirovecii: identificación de $\mathrm{P}$ jirovecii mediante tinción, PCR o histología; empiema: líquido pleural de aspecto purulento o cultivo positivo; micobacteriosis: baciloscopia o cultivo positivo o identificación de micobacteria en biopsia; sarcoma de Kaposi: hallazgos endoscópicos característicos o histología; linfoma: biopsia positiva. De no cumplir estas condiciones el diagnóstico se consideró no confirmado. Se tabularon los tratamientos efectuados, terapia antirretroviral y profilaxis específicas. Se consignaron los datos de laboratorio y la condición de egreso de los pacientes. Se revisaron registros de laboratorios y de anatomía patológica, haciendo revisión de placas y documentos en caso necesario. Dieciséis egresos que correspondieron a 14 pacientes, fueron excluidos del estudio porque no figuraba una enfermedad respiratoria como diagnóstico de egreso. Todos ellos fueron dados de alta vivos.

Método estadístico. Los diagnósticos de egreso se agruparon en tres categorías específicas: infecciosas, 
neoplásicas y misceláneas. Posteriormente se identificaron dos grupos: aquéllos con neumonía por $\mathrm{P}$ jirovecii y aquellos con otras enfermedades pulmonares distintas de la neumonía por $\mathrm{P}$ jirovecii. Se consideró como variable dependiente a la condición del paciente al egreso del hospital, esto es, vivo o fallecido. Todas las condiciones demográficas y biomédicas se consideraron variables independientes. Las variables binarias se estudiaron utilizando el test de chi cuadrado $\left(\chi^{2}\right)$. Para calcular diferencias entre promedios se utilizó el test t de Student.

En cada uno de los dos grupos, esto es, en los con neumonía por $\mathrm{P}$ jirovecii y aquéllos con otras enfermedades pulmonares distintas de la neumonía por $\mathrm{P}$ jirovecii, se realizó un estudio bivariado para identificar las variables relacionadas con la muerte de los pacientes en el hospital. Aquellas variables que alcanzaron significación estadística fueron sometidas a un estudio multivariado, utilizando un modelo de regresión logística para identificar aquellas variables biomédicas independientes que permitiesen predecir la mortalidad intrahospitalaria. Se consideró con significación estadística a un valor de $\mathrm{p} \leq 0,05$.

Para los fines de este estudio cada egreso hospitalario fue considerado como un evento diferente. Todos los cálculos estadísticos se realizaron con el programa SPSS versión 10.0 para Windows.

\section{Resultados}

En el período de 55 meses que abarcó este estudio los pacientes infectados con VIH corres- pondieron al 1,2\% del total de egresos del hospital. Se registraron 236 egresos por enfermedad pulmonar en 171 pacientes (1,4 episodios por paciente). Los datos biodemográficos se describen en la Tabla 1. La relación por sexos fue de 6,4 hombres por cada mujer. Se desconoce la orientación sexual en 53\%; 86,5\% no había accedido a terapia antirretroviral; $82 \%$ no recibía profilaxis para $\mathrm{P}$ jirovecii y $18,4 \%$ de los casos ignoraba estar infectado con VIH al momento del ingreso. La mortalidad intrahospitalaria alcanzó a 19,5\%.

Las enfermedades infecciosas fueron la principal causa de enfermedad pulmonar en esta población y constituyeron el diagnóstico de egreso en $87,2 \%$ de los casos, en 5,1\% fueron de naturaleza neoplásica y mixtas (infecciosas y neoplásicas) en 5,1\%. Solamente en 5 casos no se estableció con precisión la naturaleza de la enfermedad pulmonar (Tablas 2 y 3). Dentro de las enfermedades infecciosas, la más frecuente fue la neumonía por P jirovecii. Se planteó este diagnóstico en forma aislada o asociado a otro concomitante en 105 egresos (44,5\%). El segundo lugar correspondió a las neumonías adquiridas en la comunidad, condición que se planteó como diagnóstico de egreso exclusivo en 57 casos $(24,1 \%)$ y coexistiendo con otras enfermedades pulmonares en 6 pacientes. Las infecciones exclusivas por micobacterias se presentaron en 34 egresos $(14,4 \%)$, correspondiendo 29 casos a infección por M tuberculosis, registrándose igual número de casos de compromiso pulmonar que extrapulmonar. Las micobacterias atípicas en su forma de M avium-complex (MAC) dieron cuenta de 5 casos. Hubo 19 casos de

\section{Tabla 1. C aracterísticas biodemográficas de 236 egresos por enfermedad pulmonar en pacientes infectados con VIH}

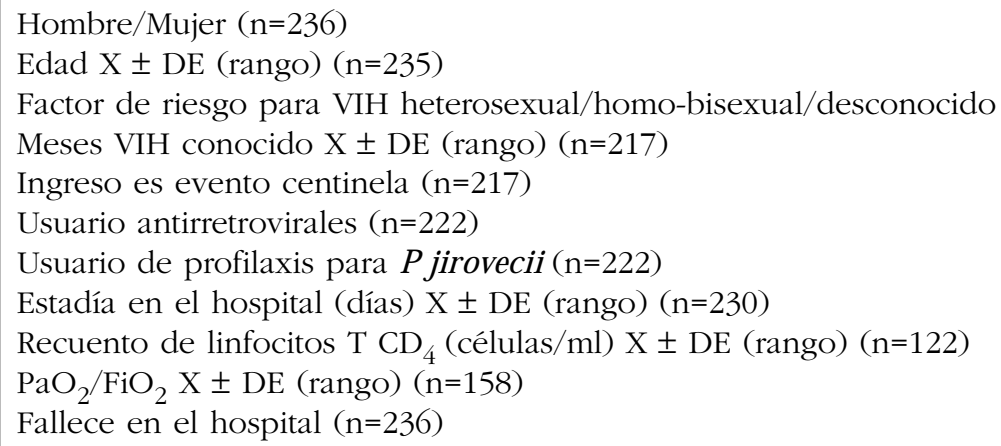

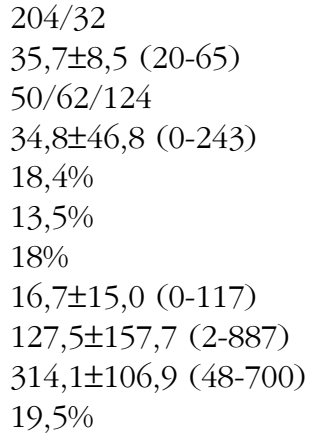


Tabla 2. Enfermedades pulmonares infecciosas en pacientes infectados con VIH

\begin{tabular}{|c|c|c|c|}
\hline \multicolumn{2}{|r|}{ Diagnóstico enfermedades infecciosas } & \multirow{2}{*}{$\frac{\text { № de casos }}{90}$} & \multirow{2}{*}{$\frac{\%}{38,1}$} \\
\hline & Micóticas & & \\
\hline & Neumonía por P jirovecii & 89 & \\
\hline & Aspergilosis pulmonar & 1 & \\
\hline \multirow[t]{5}{*}{ b) } & Bacterianas & 59 & 25 \\
\hline & Neumonía comunitaria & 55 & \\
\hline & Neumonía comunitaria estafilocócica con empiema & 2 & \\
\hline & Empiema & 1 & \\
\hline & Endocarditis infecciosa & 1 & \\
\hline \multirow[t]{10}{*}{ c) } & Micobacterianas & 34 & 14,4 \\
\hline & M tuberculosis & 29 & \\
\hline & Tuberculosis pulmonar & 13 & \\
\hline & Tuberculosis miliar & 3 & \\
\hline & Tuberculosis pulmonar y ganglionar & 5 & \\
\hline & Tuberculosis ganglionar & 4 & \\
\hline & Tuberculosis pulmonar y pleural & 2 & \\
\hline & Tuberculosis pleural & 1 & \\
\hline & Tuberculosis pleural y ganglionar & 1 & \\
\hline & M avium-complex (MAC) & 5 & \\
\hline \multirow[t]{3}{*}{ d) } & Otras infecciosas & 4 & 1,7 \\
\hline & Citomegalovirus & 1 & \\
\hline & Virosis respiratoria & 3 & \\
\hline \multirow[t]{9}{*}{ e) } & Infecciones Mixtas & 19 & 8,1 \\
\hline & Neumonía por P jirovecii + neumonía comunitaria & 5 & \\
\hline & Neumonía por P jirovecii + neumonía nosocomial & 7 & \\
\hline & Neumonía por P jirovecii + tuberculosis pulmonar & 1 & \\
\hline & Tuberculosis pleural + neumonía nosocomial & 2 & \\
\hline & Tuberculosis miliar + neumonía comunitaria & 1 & \\
\hline & Empiema + neumonía nosocomial & 1 & \\
\hline & Tuberculosis miliar + empiema + neumonía nosocomial & 1 & \\
\hline & Criptococosis pulmonar + TBC pleural + neumonía nosocomial & 1 & \\
\hline \multicolumn{2}{|c|}{ Total } & 206 & 87,2 \\
\hline
\end{tabular}

infecciones mixtas, predominando la asociación de $P$ jirovecii ya sea con neumonía comunitaria $O$ nosocomial (Tabla 2).

Hubo 14 neumonías nosocomiales, la mitad de ellas en pacientes con neumonía por $\mathrm{P}$ jirovecii y la mitad en el grupo de pacientes con otras enfermedades pulmonares distintas de la neumonía por $\mathrm{P}$ jirovecii. Se aislaron los siguientes agentes en muestras de expectoración o lavado broncoalveolar: Staphylococcus aureus meticilino resistente en 4 muestras, Acinetobacter anitratus en 3 muestras, Pseudomonas aerugino- sa en 2 muestras y Enterobacter cloacae en 1 muestra. En 5 pacientes o no se intentó o no se logró aislar un agente patógeno. Todos recibieron un esquema de tratamiento empírico con vancomicina asociada a sulperazona o a ceftazidima más amikacina. Para los casos con agente aislado el esquema antimicrobiano se ajustó según el estudio de susceptibilidad. Fallecieron 9 pacientes (Tabla 4).

Dentro de las neoplasias, el sarcoma de Kaposi fue el más frecuente, las neoplasias se asociaron a infección en 12 casos (Tabla 3). 
Tabla 3. Enfermedades pulmonares no infecciosas y mixtas en pacientes infectados con VIH

\begin{tabular}{|lcc|}
\hline Diagnóstico & № de casos & $\%$ \\
\hline 1. Enfermedades neoplásicas & 12 & 5,1 \\
Sarcoma de Kaposi & 8 & \\
Linfoma no Hodgkin del pulmón & 2 & \\
Linfoma de las serosas & 1 & \\
Cáncer pulmonar no células pequeñas & 1 & 5,1 \\
3. Enfermedades infecciosas y neoplásicas & 12 \\
S Kaposi pulmonar + tuberculosis pulmonar & 7 \\
S Kaposi pulmonar + neumonía por P jirovecii & 2 \\
S Kaposi pulmonar + neumonía nosocomial & 1 \\
Linfoma no Hodgkin pulmonar + neumonía por P jirovecii & 1 \\
Linfoma de las serosas + MAC & 1 & \\
4. Otras enfermedades & 6 & \\
Tromboembolismo pulmonar + neumonía nosocomial & 1 \\
Enfermedad pulmonar difusa no específica & 1 \\
Pleuropericarditis & 1 \\
Insuficiencia respiratoria de causa desconocida & 2 \\
Nódulos pulmonares múltiples de causa desconocida & 1 \\
Total & 30 \\
\hline
\end{tabular}

Al realizar el análisis bivariado para los 105 casos de neumonía por P jirovecii se identificaron 3 variables biomédicas asociadas significativamente con la mortalidad en el hospital: uso de esteroides, presencia de neumotórax y neumonía nosocomial. Sin embargo, al realizar el estudio multivariado, solamente la presencia de neumotórax identificó, en forma independiente y significativa, a los pacientes que fallecieron en el hospital (Tabla 4). Para el grupo de 131 pacientes con otras enfermedades pulmonares distintas de la neumonía por P jirovecii la presencia de neumonía nosocomial fue la única variable que identificó significativamente a aquellos fallecidos en el hospital. No se demostró diferencia con significación estadística para el resto de las variables estudiadas (Tabla 4).

\section{DisCusión}

La neumonía por P jirovecii fue el diagnóstico de egreso más frecuente en pacientes infectados con VIH estudiados en nuestro hospital, este hecho ya lo habíamos comunicado durante el período 1989$1997^{12}$.
En una publicación nacional, donde se revisaron las causas de hospitalización en pacientes infectados con VIH de la Fundación Arriarán, las enfermedades neumológicas fueron responsables de $29 \%$ y $38 \%$ de las hospitalizaciones durante 1999 y 2000, respectivamente. La neumonía por P jirovecii fue la enfermedad pulmonar más frecuente, seguida por las micobacteriosis y la neumonía comunitaria ${ }^{13}$.

Internacionalmente, se reconoce una disminución en la prevalencia de neumonía por $\mathrm{P}$ jirovecii en pacientes infectados con VIH, atribuido al uso de quimioprofilaxis específica y al uso extendido de la terapia antirretroviral efectiva ${ }^{5,14}$. Desgraciadamente, la población que estudiamos tiene escaso acceso a estas dos medidas terapéuticas de reconocida eficiencia. Solamente $18 \%$ de nuestra población recibía profilaxis para $\mathrm{P}$ jirovecii al momento del ingreso y 13,5\% recibía terapia antirretroviral. Estas cifras tan bajas contrastan con otras publicaciones nacionales. El 53\% de los pacientes publicados por Wolff y $\mathrm{col}^{8}$ recibía profilaxis para $\mathrm{P}$ jirovecii. En una publicación reciente de CONASI$\mathrm{DA}^{17}$ se señala que la terapia antirretroviral en el país, durante el período de este estudio, alcanzó 
Tabla 4. Variables independientes según condición al egreso del hospital en pacientes infectados con VIH con neumonía por $\mathbf{P}$ jirovecii y otras enfermedades pulmonares distintas de la neumonía por $\mathbf{P}$ jirovecii.

\begin{tabular}{|c|c|c|c|c|}
\hline \multirow[b]{2}{*}{$\begin{array}{l}\text { Condición al egreso del } \\
\text { hospital }\end{array}$} & \multicolumn{2}{|c|}{$\begin{array}{l}\text { Neumonía por } \mathrm{P} \text { jirovecii } \\
\qquad(\mathrm{n}=105)\end{array}$} & \multicolumn{2}{|c|}{$\begin{array}{l}\text { Enfermedades pulmonares } \\
\text { distintas de la neumonía } \\
\text { por P jirovecii } \\
(\mathrm{n}=131)\end{array}$} \\
\hline & $\begin{array}{l}\text { Vivo } \\
(\mathrm{n}=82)\end{array}$ & $\begin{array}{l}\text { Fallecido } \\
(n=23)\end{array}$ & $\begin{array}{c}\text { Vivo } \\
(\mathrm{n}=108)\end{array}$ & $\begin{array}{l}\text { Fallecido } \\
(n=23)\end{array}$ \\
\hline Sexo masculino & 67/82 & $22 / 23$ & $93 / 108$ & $22 / 23$ \\
\hline Edad & \multicolumn{2}{|c|}{$(n=104)$} & $\begin{array}{l}35,3 \pm 8,0 \\
(n=131)\end{array}$ & $38,9 \pm 9,6$ \\
\hline Número de hospitalizaciones & $1,2 \pm 0,5$ & $1,17 \pm 0,5$ & \multicolumn{2}{|c|}{$(n=125)$} \\
\hline Estadía en el hospital (días) & $15,7 \pm 13,3$ & $17,3 \pm 13,2$ & $\begin{array}{r}16,8 \pm 16,4 \\
(\mathrm{n}=\end{array}$ & $25)$ \\
\hline $\begin{array}{l}\text { Duración de los síntomas } \\
\text { (días) }\end{array}$ & $(n=100)$ & $\begin{array}{l}19,2 \pm 20,7 \\
00)\end{array}$ & $\begin{array}{l}29,4 \pm 48 \\
(n=108)\end{array}$ & $23,3 \pm 25,6$ \\
\hline $\begin{array}{l}\text { Diagnóstico de infección } \\
\text { VIH (meses) }\end{array}$ & \multicolumn{2}{|c|}{$(n=99)$} & \multicolumn{2}{|c|}{$(n=118)$} \\
\hline Ingreso como evento centinela & $26 / 77$ & $4 / 22$ & $8 / 100$ & $2 / 18$ \\
\hline $\begin{array}{l}\text { Recuento de linfocitos } \\
\text { T CD }\end{array}$ & \multicolumn{2}{|c|}{$(n=58)$} & \multicolumn{2}{|c|}{$\begin{array}{c}102,5 \pm 88,3 \quad 127,4 \pm 188,2 \\
(\mathrm{n}=64)\end{array}$} \\
\hline $\mathrm{PaO}_{2} / \mathrm{FiO}_{2}$ & \multicolumn{2}{|c|}{$(\mathrm{n}=76)$} & \multicolumn{2}{|c|}{$\begin{array}{c}316,8 \pm 106,4 \quad 311,8 \pm 72,9 \\
(\mathrm{n}=82)\end{array}$} \\
\hline Profilaxis para P jirovecii & $4 / 79$ & $2 / 22$ & 25/102 & 9/19 \\
\hline Uso drogas antirretrovirales & $5 / 79$ & $2 / 22$ & $18 / 102$ & $5 / 19$ \\
\hline Uso de corticoides sistémicos & $61 / 79$ & $21 / 22^{\#}$ & $13 / 102$ & $5 / 19$ \\
\hline Se realizó fibrobroncoscopia & $37 / 82$ & $11 / 23$ & $33 / 102$ & $7 / 19$ \\
\hline Diagnóstico confirmado & $32 / 80$ & $10 / 23$ & $64 / 103$ & $12 / 20$ \\
\hline Neumonía comunitaria & 4/82 & $0 / 23$ & $51 / 108$ & $8 / 23$ \\
\hline Micobacteriosis & $1 / 82$ & $1 / 23$ & $40 / 108$ & $6 / 23$ \\
\hline Neumonía nosocomial & $3 / 82$ & $4 / 23^{\#}$ & $2 / 108$ & $5 / 23^{\#}$ \\
\hline Neumotórax & $1 / 82$ & $8 / 23^{\#^{*}}$ & $1 / 107$ & $2 / 23$ \\
\hline
\end{tabular}

Los datos para las variables continuas corresponden a valores promedio \pm desviación estándar de la media, n: número de pacientes evaluados con la variable.

\#p $<0,05$ en el análisis bivariado. " p <0,05 en el análisis multivariado.

una cobertura de entre $56 \%$ y $91 \%$ de los pacientes que solicitaron el tratamiento. Esta brecha podría explicarse en parte porque $18,4 \%$ de nuestra población desconocía ser portador de la infección por VIH. Ignoramos qué porcentaje de nuestros pacientes se controlaba en centros especializados en la atención de pacientes con SIDA.

Las neumonías comunitarias son reconocidas como una causa frecuente de enfermedad pulmonar en pacientes infectados con $\mathrm{VIH}$, tanto en el medio nacional ${ }^{7,8,10,13}$ como internacional ${ }^{1,2,5,12,14}$. Correspondieron al segundo diagnóstico de egreso en nuestra serie. Un estudio multicéntrico internacional $^{15}$ estableció que las neumonías bacterianas eran cinco veces más frecuentes en personas infectadas con VIH que en aquéllas seronegativas. Se estableció, además, que los drogadictos intravenosos tenían una tasa más alta de neumonía bacteriana que los pacientes infectados con VIH que pertenecían a otros grupos de riesgo ${ }^{15}$. Se ha intentado identificar criterios de gravedad de la neumonía comunitaria en pacientes infectados con VIH, encontrándose que el shock, recuento de linfocitos $\mathrm{TCD}_{4}<100 / \mathrm{ml}$, derrame pleural, cavida- 
des y compromiso multilobar tienen una connotación de peor pronóstico ${ }^{16}$, sin embargo, se requieren otros estudios para validar estos hallazgos. La terapia antirretroviral combinada que contiene inhibidores de las proteasas, ha disminuido dramáticamente las tasas de neumonía comunitaria y neumonía por P jirovecii.

Las enfermedades producidas por micobacterias, especialmente por $\mathrm{M}$ tuberculosis, fueron una causa frecuente de egreso en esta población. Sin embargo, su incidencia descendió de $25,7 \%$ en nuestro estudio previo ${ }^{12}$ a $18,1 \%$ en la serie actual.

La presencia simultánea de dos o más causas de enfermedad pulmonar es un aspecto propio y característico de los pacientes infectados con VIH. Algunos autores han señalado que esta situación puede ocurrir en $19 \%^{18}$ y $39 \% 19$ de los casos, especialmente si se utilizan técnicas para identificar citomegalovirus. En este estudio retrospectivo, en que no se utilizaron dichas técnicas, esta situación ocurrió en 13,5\% de los casos. Las enfermedades infecciosas se asociaron entre sí en 19 casos y hubo concomitancia de enfermedades infecciosas, y no infecciosas en 13 casos. Tener esto en mente es especialmente relevante en aquellos casos en quienes, tras haber establecido un diagnóstico, se ha iniciado la terapia apropiada y no se observa recuperación. En algunos de estos casos la presencia de un copatógeno o comorbilidad es una explicación alternativa al fracaso terapéutico. Es interesante destacar que la coexistencia de dos o más causas para explicar la enfermedad pulmonar aumentó de 2,9\% en el período $1989-1997^{12}$ a $13,5 \%$ en el período examinado en el presente estudio.

Identificamos dos variables independientes asociadas significativamente con la probabilidad de fallecer en el hospital. El neumotórax, para los casos con neumonía por P jirovecii y la neumonía nosocomial, para el grupo con otras enfermedades pulmonares distintas de neumonía por $\mathrm{P}$ jirovecii.

El neumotórax es una complicación temida de la neumonía por P jirovecii. Varios estudios lo han identificado como un marcador de mal pronóstico con tasas de mortalidad que oscilan entre $50 \mathrm{y}$ $100 \% 19,20$. Teniendo esto presente es que en nuestro Instituto evitamos las biopsias transbronquiales y la ventilación mecánica con presiones positivas elevadas en pacientes con sospecha de neumonía por $\mathrm{P}$ jirovecii.
A diferencia de lo que ocurre en pacientes inmunodeprimidos no infectados con VIH, la incidencia de neumonía nosocomial ha disminuido en pacientes infectados con VIH que reciben terapia antirretroviral efectiva ${ }^{21}$. En nuestro estudio de los 14 pacientes que desarrollaron neumonía nosocomial, 9 fallecieron. Otros autores han establecido que la presencia de neumonía nosocomial aumenta en más de 5 veces el riesgo de morir en pacientes hospitalizados e infectados con $\mathrm{VIH}^{21}$.

Queremos destacar que la principal limitación de este estudio es su carácter descriptivo y retrospectivo, en él no se ha empleado una metodología estándar para el estudio de los pacientes, sino que la práctica clínica habitual del servicio de neumología de nuestro Instituto. Es por ello, que desconocemos el número de pacientes afectados por infecciones virales como influenza y citomegalovirus o por patógenos respiratorios "atípicos" que no son estudiados rutinariamente en nuestros medio. La verdadera incidencia de infección por MAC pudiera ser mayor a la aquí descrita por cuanto los hemocultivos para micobacterias se solicitaron solamente en casos seleccionados. El escaso número de casos con datos completos en una serie de variables nos ha impedido identificar la relación existente entre otros factores, tales como el recuento de linfocitos $\mathrm{T} \mathrm{CD}_{4}$, la $\mathrm{PaO}_{2} / \mathrm{Fi}_{\mathrm{i}} \mathrm{O}_{2}$ baja, el tiempo de evolución de la infección por VIH y la probabilidad de fallecer de los pacientes. Todos éstos han sido señalados por otros autores como factores pronósticos en las enfermedades pulmonares asociadas a la infección por VIH. Pensamos que el hecho de que éste haya sido un estudio retrospectivo nos ha impedido identificar estos marcadores.

Concluimos que esta serie de pacientes chilenos infectados con VIH presenta una amplia variedad de enfermedades pulmonares dentro de las cuales predominan las enfermedades infecciosas. Dentro de éstas la neumonía por P jirovecii, las neumonías comunitarias y las enfermedades provocadas por micobacterias son las más frecuentes. Estos pacientes tienen una mortalidad intrahospitalaria elevada, falleciendo uno de cada 5. Dos variables independientes identificaron significativamente a aquéllos que fallecieron en el hospital: presencia de neumotórax en los con neumonía por $\mathrm{P}$ jirovecii y neumonía nosocomial en aquellos con otras enfermedades pulmonares distintas de la neumonía por P jirovecii. 


\section{REFERENCIAS}

1. Murray J, Felton C, Garay S et al. Pulmonary complications of the acquired immunodeficiency syndrome. N Engl J Med 1984; 310: 1682-8.

2. Miller R. HIV-associated respiratory diseases. Lancet 1996; 348: 307-12.

3. Afessa B, Greaves W, Green W, Oloponeia L, Saxinger C, Frederick W. Autopsy findings in HIVinfected inner-city patients. J Acquir Immune Defic Syndr 1992; 5: 132-6.

4. Masliah E, DeTeresa R, Mallory M, Hansen L. Changes in pathological findings at autopsy in AIDS cases for the last 15 years. AIDS 2000; 14 : 69-74.

5. Rosen M. Overview of pulmonary complications. Clinics in Chest Medicine 1996; 17: 621-31.

6. Batungwanayo J, Taelman H, Lucas S, Bogaerts J, Alard D, Kagame A et al. Pulmonary disease associated with the human immunodeficiency virus in Kigali, Rwanda. A fiberoptic bronchoscopic study of 111 cases of undetermined etiology. Am J Respir Crit Care Med 1994; 149: 1591-6.

7. Wolff M, Northland R, Segovia J, Beltrán C, Valdés M, Albornoz M et al. Características clínicas e historia natural de la infección por virus de inmunodeficiencia humana. Estudio en una población chilena atendida en un centro piloto multiprofesional. Rev Méd Chile 1995; 123: 61-73.

8. Wolff M, Diomedi A, Morales O, Bidart T, Dabanch J, Bustamante C et al. Seguimiento prospectivo de una población infectada por VIH con y sin posibilidades de terapia anti-retroviral: impacto en sobrevida y complicaciones. Rev Méd Chile 2001; 129: 886-94.

9. SchönfFeldt P, Chernilo S, Trujillo S, SchönfFeldt M, Mamani R. Linfoma de las serosas asociado a Mycobacterium avium intracelular en un paciente con SIDA. Caso clínico. Rev Chil Enferm Respir 2002; 18: 267

10. Niederman MS, Mandell LA, Anzueto A, Bass JB, Broughton WA, Campbell GD et al. Guidelines for the management of adults with communityacquired pneumonia. Diagnosis, assessment of severity, antimicrobial therapy and prevention. Am J Respir Crit Care Med 2001; 163: 1730-54.

11. Hospital-acquired pneumonia in adults: diagnosis, assessment of severity, initial antimicrobial therapy, and preventive strategies. A consensus statement, American Thoracic Society, November 1995. Am J Respir Crit Care Med 1996; 153: 171125.

12. Zagolín M, Morales P, Chernilo S. Compromiso pulmonar en pacientes VIH (+), experiencia del INERCYT, 1989-1997. Rev Chil Enferm Respir 1997; 13: 245.

13. Ministerio de Salud, Comisión Nacional del SIDA. Evaluación del impacto de una política pública: Ampliación del acceso a terapia antirretroviral en Chile. Rev Chil Infect 2003; 20: 277-84.

14. Hoover DR, SaAh AJ, Bacellar H, Phair J, Detels R, Anderson R et al. Clinical manifestations of AIDS in the era of Pneumocystis prophylaxis. N Eng J Med 1993; 329: 1922-6.

15. Hirschtick RE, Glassroth J, Jordan MC, Wilcosky TC, Wallace JM, Kvale PA et al. Bacterial pneumonia in persons infected with the human immunodeficiency virus. N Eng J Med 1995; 333: 845-51.

16. Cordero E, Pachon J, Rivero A, Girón JA, GómezMateos J, Merino M et al. Community-acquired bacterial pneumonia in human immunodeficiency virus-infected patients: validation of severity criteria. The Grupo Andaluz para el Estudio de las Enfermedades Infecciosas. Am J Respir Crit Care Med 2000; 162: 2063-8.

17. Comisión Nacional de SIDA-CONASIDA. Ministerio de Salud. Panorama epidemiológico de la infección por VIH/SIDA en Chile-Año 2001. Rev Chil Infect 2002; 19: 245-61.

18. Fernández A, Pulido F, Peña JM, Sanz M, Lorenzo A, GonzÁlez J ET AL. Factores pronósticos de mortalidad durante el episodio de neumonía por Pneumocystis carinii en pacientes con infección por VIH. Rev Clin Esp 2002; 202: 416-20.

19. Stover DE, White DA, Romano PA, Gellene RA, Robeson WA. Spectrum of pulmonary diseases associated with the acquired immune deficiency syndrome. Am J Med 1985; 429-37.

20. Levine SJ. Pneumocystis carinii Pulmonary Complications of HIV infection. Clinics in Chest Med 1996; 17: 665-95.

21. Tumbarello M, Tacconelli E, de Gaetano Donati K, Bertagnolio S, Cataldo M, Pirronti T et al. Nosocomial bacterial pneumonia in human immunodeficiency virus infected subjects: incidence, risk factors and outcome. Eur Respir J 2001; 636-40. 